\title{
Drogue et politique: le cas panaméen
}

\section{Gilles Bataillon}

\section{(2) OpenEdition}

\section{Journals}

\section{Édition électronique}

URL : http://journals.openedition.org/conflits/115

DOI : 10.4000/conflits. 115

ISSN : $1777-5345$

Éditeur :

CCLS - Centre d'études sur les conflits lilberté et sécurité, L'Harmattan

\section{Édition imprimée}

Date de publication : 17 octobre 1991

ISSN : 1157-996X

\section{Référence électronique}

Gilles Bataillon, « Drogue et politique: le cas panaméen », Cultures \& Conflits [En ligne], 03 | automne 1991, mis en ligne le 31 décembre 2002, consulté le 30 mars 2021. URL : http:// journals.openedition.org/conflits/115; DOI : https://doi.org/10.4000/conflits.115

Ce document a été généré automatiquement le 30 mars 2021.

Creative Commons License 


\title{
Drogue et politique: le cas panaméen
}

\author{
Gilles Bataillon
}

Depuis la fin des années 70, Panama est devenu un lieu de transit pour la cocaïne à destination des Etats-Unis, ainsi qu'une place financière susceptible d'accueillir les bénéfices provenant du trafic de drogue, sans que ces activités apparaissent illégitimes aux yeux de la plupart des Panaméens. De plus, bon nombre de ces activités ont souvent été connues et soumises au contrôle des membres des Forces de défense panaméennes, sous le gouvernement du général Torrijos (1968-1981) puis sous le gouvernement indirect de ses successeurs à la tête de l'institution militaire, les généraux Paredes (1981-1983) et le général Noriega (1983-1989). Si cet afflux de ressources, lié au boom du trafic de coca-cocaïne, a contribué à la prospérité des banques panaméennes et à l'enrichissement de nombreux militaires, ce phénomène est aussi entré en interaction avec les affrontements politiques panaméens. En effet, en 1983, fort des ressources procurées par ce trafic et de son nouveau statut de commandant en chef des armées, Noriega entreprenait tout à la fois, de contrôler très systématiquement la plupart des activités liées au trafic de drogue et d'utiliser les ressources procurées par ces activités pour modifier le système politique panaméen, en mettant fin aux traditionnels arrangements entre les militaires et les autres concurrents pour le pouvoir (ce qui devait déclencher un tollé chez ceux-ci). Enfin, dans un dernier temps, en 1987 la crise politique liée aux visées hégémoniques de Noriega connaissait un rebondissement dû à son inculpation pour trafic de drogue par un jury de Miami. En effet, dès lors les EtatsUnis qui avaient longtemps toléré les trafics de la Garde nationale et les prétentions dictatoriales de Noriega changeaient d'attitude du tout au tout. Mettant l'accent sur leur volonté de lutter contre le narco-trafic et de promouvoir la démocratie, ils encourageaient l'opposition et les militaires hostiles à Noriega à le renverser, et pour finir, pratiquaient une intervention directe - Just Cause, décembre 1989 - qui conduisait à l'arrestation de Noriega, à la dissolution des forces armées et à l'installation d'un gouvernement civil. 
2 Saisir les réalités liées au trafic de drogue ainsi que les interactions entre ce trafic et l'évolution politique panaméenne, tels sont les propos de cette étude. La première partie de ce travail portera sur le monde du narco-trafic. On commencera par en repérer les principaux protagonistes, on s'interrogera sur l'existence d'un milieu spécifique doté d'une identité et de modes d'action propres et on analysera les relations de ces acteurs avec les normes de la société panaméenne. La seconde partie portera sur les visées des principaux acteurs liés à ce trafic. Pour ce faire, on évoquera d'abord le contexte panaméen et le rôle qu'y jouent les militaires pour analyser par la suite les actions de leurs deux principales têtes de file, Torrijos et Noriega, et leur inscription dans le contexte panaméen. Une troisième et dernière partie sera consacrée à ce que la plupart des observateurs ont dénommé "la crise panaméenne" (1987-1989). On cherchera à saisir les modifications apportées au contexte panaméen par l'utilisation faite par Noriega des ressources provenant des multiples trafics contrôlés par les militaires pour modifier les règles du jeu propres à la société panaméenne, et ce faisant, favoriser l'apparition de tensions nouvelles qui rendront possible l'opération Just Cause.

Les acteurs du narco-trafic

3 Inexistence d'un milieu spécifique, difficulté à distinguer le trafic de drogue de multiples autres activités à la limite de l'illégalité, ancienneté de ces activités, légitimité de ces modes d'enrichissement, telles semblent être les caractéristiques recouvertes par le trafic de drogue à Panama.

L'absence d'un milieu spécifique

4 Sans doute ne saurait-on passer sous silence l'existence de réseaux spécialisés dans le trafic de drogue comme l'ont prouvé certaines dépositions faites devant le Congrès ou le Sénat des États-Unis par d'anciens complices de Noriega dans le narco-trafic. Ainsi on a la preuve de l'existence d'opérations montées au plus haut niveau entre des narcos colombiens et Noriega, ou entre ce dernier et un trafiquant de marijuana nordaméricain - Stephen Kalish; de plus on sait que certaines banques ou certains cabinets d'affaires se faits une spécialité du blanchiment de narco-dollars(1). Il convient toutefois de souligner que le gros des actions liées au c de drogue sont réalisées par des acteurs collectifs ou individuels pour lesquels elles restent des activités occasionnelles qui n'entraînent jamais une redéfinition de leurs statuts. On sait ainsi que les militaires panaméens ont joué un rôle clé, et ce, à un double niveau. Grâce aux informations fournies par ses services de renseignements, l'institution militaire a systématiquement prélevé sa dîme sur les trafiquants venus déposer leurs bénéfices dans les banques panaméennes, et a taxé ceux d'entre eux utilisant le territoire panaméen pour faire transiter leurs marchandises. A côté de ces ponctions destinées à alimenter, dès la fin des années 70, les caisses noires des forces armées, on constate une activité de racket à des fins d'enrichissement personnel, pratiquée au jour le jour par les militaires à l'encontre des trafiquants. Celle-ci, loin d'être l'apanage des officiers supérieurs, comme le prouve amplement l'exemple de Noriega, était aussi le fait d'hommes occupant des rangs subalternes(2).

5 De même, à en croire plusieurs documents DEA(3), la plupart des 110 banques panaméennes auraient à un moment ou un autre accepté de faire transiter tous les ans, entre 1,2 milliard et 2,2 milliards de narco-dollars par leurs guichets. Quelques dix banques se seraient spécialisées dans cette activité, parmi lesquelles la filiale panaméenne de la BCCI, le Banco institucional patria, et la First interamericas bank... 
tandis que d'autres, sans s'en faire une spécialité, auraient volontiers accueilli les capitaux douteux, et qu'enfin, jusqu'en 1937, les banques les plus responsables - la BNP, la Dresden banq et l'Union des banques suisses... - se seraient, quant à elles, bornées à refuser les dépôts en liquide supérieurs à 100000 dollars.

On constate le même phénomène au niveau des cabinets d'affaires. Certains avaient pour principale activité la vente de sociétés-écran aux narco-trafiquants, d'autres se contentaient de réaliser occasionnellement ce genre d'opérations. Ainsi l'actuel président de la République panaméenne, Guillermo Endara, a été accusé d'avoir détenu $2 \%$ des actions d'une banque en relation avec les trafiquants colombiens, et a reconnu avoir contribué avec ses associés à créer une vingtaine de sociétés destinées à accueillir des narco-dollars. De même le porte-parole de son gouvernement est accusé d'avoir participé à une opération de recyclage portant sur un million de narco-dollars(4).

7 Le rôle de premier plan joué par les militaires dans les transactions avec les narcotrafiquants ne saurait conduire à penser que cette activité a été l'apanage d'un clan politique formé par les forces armées, les membres de leur parti politique - le Parti révolutionnaire démocratique - et les hommes d'affaires proches de ces deux entités. $\mathrm{Si}$ les militaires et leurs proches sont sans doute les plus impliqués par le nombre et le volume des transactions auxquels ils ont participé, il n'est peu ou pas d'entités de la société panaméenne qui ne se soient trouvées liées d'une façon ou d'une autre à ces affaires comme le prouve l'exemple de Guillermo Endara.

Un trafic parmi d'autres

8 Loin de constituer une activité en rupture avec les autres opérations réalisées par les militaires et le monde des affaires, les relations avec les narco-trafiquants semblent s'inscrire en continuité avec des activités plus anciennes.

En effet, l'essor de la zone franche de Colón, créée en 1951, s'est accompagné du développement d'une contrebande protéiforme à destination de Panama, de la Colombie, du Venezuela, puis de Cuba, à la suite du rétablissement des relations diplomatiques avec ce pays (1974). Dès la fin des années 60, les militaires sont partie prenante de ces différentes affaires. Certaines sociétés-écran sont créées par ceux-ci à des fins strictement privées, d'autres - telle Transit S.A. - le sont dans le cadre de l'institution militaire afin d'alimenter sa caisse noire. De plus, nombre de compagnies installées à Colón versent régulièrement des "salaires" aux officiers panaméens ou offrent des pots de vin lors d'opérations de contrebande. Ces multiples affaires ne sont pas sans lien avec des opérations réalisées par les services secrets : vente de passeports à leurs homologues chinois de Taiwan ou aux Israéliens et au Nord-américains au début des années 70 ; trafic d'armes à destination des Sandinistes, de la guérilla salvadorienne puis des Contras ou des Argentins lors de la guerre des Malouines. Il convient d'ajouter à ces revenus alimentant la caisse noire des forces armées ceux versés par les EtatsUnis au titre de la collaboration des services de renseignements panaméens avec le Pentagone et la CIA. Notons que, par-delà la diversité de leurs origines, les revenus générés par les multiples activités, extra-militaires ou paramilitaires, des membres de la Garde nationale alimentent tous au même titre la caisse noire des forces armées. C'est dire qu'à partir des années 70 , les revenus procurés par la participation au narcotrafic deviennent indissociables de ceux issus du trafic d'armes, de la contrebande d'appareils électroménagers ou de l'aide militaire nord-américaine. Ainsi quelle que soit la spécificité du trafic de drogue au regard de la législation internationale, il importe de noter que pour ces acteurs de premier plan que sont les militaires, ce trafic 
n'est qu'une affaire parmi d'autres dont certaines sont légales - l'échange de services avec les Américains - d'autres au marge de la légalité les "dons" des compagnies opérant dans la zone franche-, et d'autres illégales tels les multiples exemples de concussion, de prévarication ou de racket(5).

10 On constate un phénomène analogue dans le monde de la banque et des cabinets d'affaires. Les narco-dollars sont loin d'être les premiers capitaux douteux à être blanchis par les circuits financiers panaméens. Avant même la création du Centre financier international (1970), les entreprises de la zone franche ont servi de sociétésécran aussi bien aux multinationales pratiquant l'évasion fiscale qu'à celles pratiquant l'évasion de capitaux. Ces sociétés ont aussi fait bon accueil à des capitaux provenant du trafic d'armes ou aux pétrodollars au lendemain de la crise pétrolières.

11 Aussi, d'un point de vue sociologique convient-il, tout spécialement dans le cas des militaires, de ne pas raisonner en terme de "trafic de drogue" mais de "trafic", sachant que ce terme recouvre des opérations diverses qui sont au même titre source de revenus pour l'ensemble des acteurs qui y sont impliqués. De plus il importe de mettre en avant un autre phénomène. Quel que soit le volume de ces différentes transactions et l'importance des revenus qu'elles procurent aux acteurs panaméens impliqués, la participation à ces opérations n'entraîne pas une redéfinition de l'identité de ces acteurs.

Des activités légitimes

12 Eu égard aux normes explicites et implicites de la société panaméenne, l'ensemble de ces activités, quels que soient leurs protagonistes, apparaissent comme des modes d'enrichissement licites. Le cas est particulièrement net pour les banquiers et les hommes d'affaires, il ne l'est pas moins pour les militaires.

13 Ainsi, le blanchiment des capitaux est une pratique socialement acceptée par les banquiers et les hommes d'affaires. Il faudra attendre 1986 pour qu'une loi interdise explicitement aux banques et aux cabinets d'affaires d'accepter des dépôts provenant des bénéfices réalisés par des trafiquants de drogue. Ces dispositions légales seront toutefois loin d'être acceptées comme le prouvent les déclarations de maints responsables politiques et hommes d'affaires panaméens à l'occasion de la ratification du traité d'assistance légale mutuelle avec les Etats-Unis'. Le président Guillermo Endara déclarait pour justifier sa réticence à accepter la levée du secret bancaire en cas d'enquêtes sur le trafic de drogue: "Nous n'allons pas assassiner notre système bancaire". Le président du Collège des avocats affirmait quant à lui que ce traité "mettait en danger la vie de nombreux juristes panaméens dont la plupart des revenus proviennent de la création de sociétés panaméennes". Le vice-président de l'association des banquiers panaméens proclamait $\mathrm{H}$ dans la banque on a toujours pensé qu'un transfert provenant d'une autre banque pouvait être considéré comme insoupçonnable"(9).

14 La concussion liée au trafic réalisé par les militaires fait l'objet de la même tolérance et s'inscrit en continuité avec une représentation qui fait de la politique et de l'accès à des fonctions officielles autant de voies légitimes de l'enrichissement personnel. Ainsi la fortune de Noriega et de ses proches, celles de nombreux autres officiers, loin d'être stigmatisées sont considérées comme allant de soi et sont légitimées par ces précédents illustres qu'ont été l'enrichissement du major Remon dans les années 50 et celui du leader populiste Arnulfo Arias. Le premier instituera la pratique des dons des compagnies opérant dans la zone franche aux officiers de la Garde nationale ainsi que 
la prise en main des réseaux de prostitution par ces mêmes officiers. Le second sera triomphalement réélu en 1968 après avoir été accusé d'avoir détourné quelque 7 millions de dollars lorsqu'il était président de la République en 1949. Cette tolérance est à la fois le fait des vieilles familles patriciennes libérales et des secteurs populaires. Les unes ont toujours fait bon accueil, même à l'époque où elles formaient une oligarchie, aux nouveaux riches quelle que soit l'origine de leurs fortunes. Les autres ont vu dans l'ascension sociale du major Remon, puis dans celle du général Noriega, tous deux issus de milieux modestes, autant d'exemples de réussite auxquelles elles pouvaient s'identifier(10).

Les Etats-Unis qui jouent un véritable rôle de futur vis-à-vis de la nation panaméenne et tout spécialement à l'égard des forces armées, ne vont pas avoir une autre attitude. Guidés par le soucis de garantir leurs intérêts stratégiques, ils ne se contenteront pas de tolérer ces pratiques, mais seront des partenaires des services de renseignements panaméens dans nombre de leurs opérations extra-militaires. En effet, si sous le gouvernement Carter, les forces armées et les services de renseignements des EtatsUnis collaborent activement avec la Garde, ils ne participent à aucun des trafics des membres des forces armées panaméennes, et se contentent parfois de protéger certains Panaméens sous le coup d'inculpation de la justice américaine". En revanche, tout au long du premier mandat Reagan puis jusqu'en 1988 lors de son second mandat, différentes institutions nord-américaines vont utiliser les savoir-faire mis en oeuvre par les Panaméens dans leurs transactions illicites. Les hommes du G2 panaméen seront les artisans de l'acheminement de toute une partie de la logistique fournie aux Contras, ils feront transiter des Exocets français aux mains des Américains vers l'Argentine durant la guerre des Malouines(12). Cette capacité à utiliser ce type de compétence des militaires panaméens a d'ailleurs un précédent puisque, durant la Deuxième Guerre mondiale, l'organisation des "loisirs" des soldats américains opérant dans le Pacifique seront organisés en étroite collaboration avec les officiers de la Garde nationale qui contrôlent les réseaux de prostitution du pays(13).

Drogue, ressources de pouvoir et politique : de Torrijos à Noriega

16 La situation politique panaméenne se caractérise par les traits suivant: une hétérogénéité des acteurs politiques où on trouve des partis aux structures les plus diverses (parti de personnalistes comme les Panaménéistes d'Arnulfo Arias, parti de masse comme le Parti démocrate chrétien, parti né à l'ombre de l'État comme le Parti révolutionnaire démocratique, parti héritier de la vieille division libéro-conservateur), des organisations socio-professionnelles comme le Conseil national de l'entreprise privée (CONEP), des institutions militaires, églises, mouvements étudiants et des réseaux clientélistes; une prédominance de fait des militaires sur les autres acteurs; une inexistence de sources de pouvoir (élection, coup d'État) s'imposant légitimement pour désigner un gouvernement; des arrangements et négociations ad hoc entre les différentes forces socio-politiques; une politique faite par un petit nombre et un droit d'immixtion des Etats-Unis"(14).

17 A l'image de ce qu'a mis en lumière Charles Anderson dans ses études consacrées au système politique latino-américain(15), la politique est conçue par les acteurs panaméens comme un processus de manipulation ou de négociation entre des "concurrents pour le pouvoir" dont les ressources font l'objet d'une appréciation et d'une reconnaissance réciproque afin de parvenir à un accord négocié au sommet. Il convient de noter que ces ressources de pouvoir sont tout aussi diverses que les acteurs 
que nous évoquions. En effet, celles-ci sont aussi bien, la capacité à mobiliser des électeurs lors d'un scrutin, que celles d'organiser une manifestation de rue, une émeute ou un coup d'état, que le contrôle de ressources économiques, que des appuis internationaux et au premier chef celui des Etats-Unis. C'est dire qu'à cet égard les élections, quelle que soit la révérence affichée pour la légitimité démocratique ne sont qu'une ressource ponctuelle parmi d'autres et que les électeurs sont le plus souvent convoqués pour ratifier un accord conclu au sommet entre les têtes de file des différents groupes de concurrents. Notons encore que si ce système admet l'entrée en lice de nouveaux prétendants, pour peu qu'ils fassent preuve de leurs capacités de pouvoir il ne permet en aucun cas l'élimination de ceux dont les ressources viendraient à diminuer ou disparaître. Une autre règle tacite veut qu'au cas où un nouveau prétendant veuille éliminer un concurrent plus ancien du cercle des négociations, celui-ci se ligue aux autres pour exclure le nouveau venu jusqu'à ce qu'il accepte cette quasi-impossibilité de réduire le nombre des concurrents.

18 Eu égard à la situation politique panaméenne, les trafics réalisés par les militaires méritent une attention toute particulière. A la différence de ceux mettant en cause banquiers ou avocats d'affaires qui intéressent avant tout une collection d'individus atomisés, pour la plupart sans rôle ni prétention politique, les trafics réalisés à l'ombre des forces armées apparaissent à la fois comme favorisés par le contexte panaméen, comme mettant en cause un acteur politique central, et enfin comme susceptibles de faire apparaître de nouveaux lieux de tension et d'instabilité, c'est-à-dire autant d'éléments définissant un nouveau contexte. Notons de surcroît que les revenus procurés par les trafics militaires sont autant de ressources à disposition de leur institution. Aussi convient-il de s'interroger sur l'usage fait de ces ressources, sous le commandement de Torrijos puis sous celui de Noriega et de tenter de mettre à jour les éventuelles modifications apportées au contexte politique par ces utilisations.

Torrijos ou la consolidation d'un concurrent pour le pouvoir

Inexistantes ou presque à l'époque où Torrijos et les militaires s'emparent du pouvoir, les ressources liées aux trafics de la Garde nationale vont se multiplier dès lors que celle-ci est à la tête de l'État: phénomène qui permettra à Torrijos de consolider sa situation, d'exercer durablement le pouvoir et de renforcer les positions des forces armées dans le système politique. En effet, le coup d'État de 1968 et la destitution du président élu - Arnulfo Arias répondent aux prétentions de ce dernier d'exclure les militaires, et plus spécialement les officiers supérieurs, du cercle des négociations politiques auxquelles ils avaient accédé à la suite de la transformation de la Garde corps au départ purement policier - en une institution apte à accomplir à la fois des tâches policières et militaires; transformation opérée par le colonel Remon durant la Seconde Guerre mondiale. De plus, loin d'être réalisé sans le soutien de secteurs civils, ce coup de force reçoit au contraire le soutien de secteurs libéraux hostiles au Parti panaménéiste, tandis que les Etats-Unis traditionnellement méfiants à l'égard d'Arnulfo Arias restent neutres(16).

20 Aussi est-ce une fois parvenus au pouvoir que les militaires vont voir leurs ressources se multiplier et que, fort de cette situation éminemment favorable, Torrijos va systématiquement chercher à renforcer sa situation de concurrent pour le pouvoir tant au niveau des forces armées qu'au niveau national. Sa capacité à laisser toute initiative à ses subordonnés pour s'enrichir par différents trafics et à utiliser les revenus procurés par ces trafics pour, d'une part, alimenter un parti politique à sa dévotion, le 
Parti révolutionnaire démocratique, et pour, d'autre part, recueillir les faveurs de certaines personnalités politiques nationales ou internationales, en s'assurant de l'appui de certains secteurs sociaux par des faveurs clientélistes, s'inscrivent dans le cadre de cette stratégie. Son influence grandissante au sein de la Garde nationale puis du Parti révolutionnaire démocratique excède celle habituellement dévolue à un chef de corps, par le jeu des principes de commandement et d'obéissance propre aux structures militaires et celles reconnues à un leader politique dans un parti de masse bureaucratiquement organisé. La hiérarchie militaire est partiellement court-circuitée par le commandant en chef au profit de relations verticales avec ces subordonnés, les règles de l'avancement sont remaniées par le contrôle personnel de Torrijos sur toutes les nominations, tandis que les instances propres au Parti révolutionnaire démocratique sont soumises à ses moindres desiderata.

21 Paradoxalement, la montée en puissance des militaires au sein de la société panaméenne et celle de Torrijos au sein de ces deux entités ne va générer que des tensions ponctuelles mais en aucun cas des tensions durables. En effet, si au début des années 70 , les militaires font à plusieurs reprises usage de la violence à l'encontre de leurs concurrents - arrestations multiples, harcèlement de la presse, mise au pas de tous les mouvements socio-professionnels indépendants, dissolution de l'Assemblée nationale et interdiction des partis politiques jusqu'en 1978 avec la création en contrepartie d'une assemblée des Correjimientos formée de représentants désignés par la Garde - ces violences s'accompagnent néanmoins d'une capacité à négocier avec ceux-là même qu'ils persécutent et à en coopter certains. Ainsi par exemple si les partis n'ont plus droit de cité, plusieurs de leurs chefs de file n'en sont pas moins en étroit contact avec les militaires et ils élaborent en commun le projet de développement du centre financier international, tout comme ils jettent les premières bases de la renégociation de traité du Canal avec les Etats-Unis. De même quels que soient les harangues nationalistes de Torrijos, capable de renouer avec Cuba en lui offrant la possibilité, grâce à de multiples sociétés-écran de tourner l'embargo nord-américain, ce dernier saura toujours composer avec les Etats-Unis, notamment en acceptant les amendements introduits unilatéralement par le Sénat lors de la conclusion du nouvel accord sur le Canal: amendements qui, rappelons-le, garantissent aux Etats-Unis le droit d'intervenir sur tout le territoire panaméen pour protéger le canal(17).

On distingue maints autres exemples de cette capacité à renforcer cette position tout en faisant la part du feu. Ainsi en 1973, suite à des incidents survenus en réaction aux agissements du gouverneur communiste de la province de Chiriqui, Torrijos choisit-il de limoger celui-ci et ses plus proches collaborateurs et de donner satisfaction aux représentants du CONEP et de l'Église. De même en 1978, face à la conjonction d'une conjoncture économique défavorable, aux critiques de l'opposition et aux sollicitations $\mathrm{du}$ gouvernement Carter, Torrijos procède à une indéniable ouverture politique en amendant la constitution promulguée par l'Assemblée des Correjimientos. Il renonce à occuper le devant de la scène politique et nomme un président de la République civil, il libéralise la loi sur le droit d'association et en 1980 convoque à des élections semicompétitives débouchant sur un Conseil national de législation chargé de modifier la constitution. Enfin il promet des élections libres(19) en 1984.

23 La conduite de Torrijos au sein des forces armées obéit au même principe. Nous avons déjà souligné sa capacité à laisser quartier libre à ces subordonnés pour s'enrichir, reste à souligner ses grandes lignes de conduite à l'égard de ses pairs au sein de l'État major. 
Quelle qu'y soit sa prédominance, les marchandages semblent constants avec ses rivaux et si ceux-ci sont parfois éconduits, ils sont au mieux mis sur des voies de garage, mais jamais éliminés par des mises à la retraite anticipée et continuent toujours de bénéficier, au même titre que les fidèles, des largesses procurées par les trafics.

En bref, l'accès au pouvoir de la Garde et de leur commandant en chef en 1968 s'inscrit dans le respect des règles du modèle mis à jour par Anderson dans ces études du système politique latino-américain. Si le règne des militaires a été consubstantiel de la multiplication de leurs ressources de pouvoir, ce mouvement n'a pourtant pas contribué à faire apparaître de nouveaux lieux de tension, ce pour deux raisons. Torrijos et les militaires ont toujours su contrebalancer leur montée en puissance en multipliant les arrangements avec leurs concurrents et en se refusant à éliminer les plus faibles d'entre eux, ils ont respecté ce faisant les règles de la société panaméenne. De plus, le commandant en chef des forces armées a fait preuve du respect des mêmes normes à l'intérieur de l'institution militaire. L'instabilité propre aux transactions sur lesquelles reposent les revenus des forces armées en tant qu'institution et collection d'individus ayant accédé à l'aisance matérielle, et dans certains cas, à la fortune est en revanche grosse de tensions futures dans la mesure où ces transactions sont plus que sensibles aux bouleversements de la conjoncture internationale.

Noriega, de la volonté de régner seul à la multiplication de lieux de tensions

A la différence de ce que l'on a pu constater en examinant l'utilisation des ressources procurées par les multiples trafics menés par les forces armées à l'époque de Torrijos, les pratiques de Noriega vont, à la fois indiscutablement renforcer son pouvoir sur la Garde nationale et sur la société panaméenne, ainsi que la puissance de la première sur la seconde. Mais ce double mouvement sera coextensif d'une multiplication des tensions au sein de l'armée elle-même, ainsi que dans les rapports que celle-ci entretient avec la société panaméenne et la puissance tutélaire que sont les États-Unis.

Quelques clichés et un bref survol des six années durant lesquelles Noriega siègera à la tête des Forces de défense panaméenne suffisent à attester de l'accroissement des ressources procurées, à titre individuel et collectif, aux militaires par les trafics réalisés sur leur continent ainsi que l'accroissement sans précédent de leur puissance politique.

Le doublement des salaires des officiers qui avait cours sous Torrijos cède la place à des rémunérations beaucoup plus élevées versées directement par Noriega, rémunérations qui s'élèvent à quelque 20000 dollars par mois pour les officiers supérieurs(20) tandis que Noriega amasse à titre personnel une fortune de quelque 200 millions de dollars(21). Cet enrichissement va de pair avec une capacité grandissante de Noriega à imposer ses volontés aux militaires et, fort de cet appui, à faire de même avec les Panaméens. En effet, il invitera son prédécesseur à la tête des forces armées à lui céder la place avant de limoger son éventuel successeur et nombre d'autres opposants internes, de plus il créera des structures court-circuitant la hiérarchie au sein des forces armées. Alors que l'ensemble du sous-continent voit se multiplier un retour aux casernes des militaires et des gouvernements civils démocratiquement élus, les Forces de défense panaméennes feront non seulement élire frauduleusement leur candidat à la présidence de la République en 1984 mais n'hésiteront pas à le destituer dès qu'il fera preuve d'indépendance à leur égard, le remplaçant par un nouvel homme de confiance qui connaîtra bientôt le même sort. Pour finir elles annuleront purement et simplement, comme on s'en souvient, les résultats du scrutin de 1989 qui leur étaient défavorables. Ces images invitent donc à souligner combien chacun des renforcements 
de la puissance de Noriega a été de pair avec l'apparition de nouveaux lieux d'instabilité(22).

La première source de tension tient à la nature même des trafics qui vont de la concussion à la contrebande, sans oublier le narco-trafic. A la différence de ce que l'on pouvait constater sous Torrijos, bon nombre de transactions réalisées par les forces armées posent problème dans les relations avec les Etats-Unis. La capacité de Cuba à contourner l'embargo américain grâce à la multiplication de sociétés-écran panaméennes dans la zone franche est l'objet de nombreuses inquiétudes du gouvernement Reagan. De plus, les facilités procurées aux trafiquants de drogue ne suscitent tout d'abord que l'hostilité de certaines personnalités politiques américaines avant de devenir une véritable pierre d'achoppement dans les relations entre les deux pays, quand un jury de Miami aura inculpé le commandant en chef des Forces de défense panaméennes pour trafic de drogue. Ce trafic d'armes longtemps encouragé notamment en direction des Contras vient à faire l'objet de nombreuses controverses dans la mesure où il alimente les guérillas salvadorienne et colombienne ainsi que certaines milices privées liées aux narcos colombiens.

Un second foyer d'instabilité apparaît au sein de l'armée, au lendemain de l'accès de Noriega au poste de commandant en chef. Cette instabilité s'enracine à deux niveaux. Si la verticalisation de tous ces trafics sous l'autorité de Noriega et des hommes des services secrets permet d'offrir des gratifications plus importantes à un plus grand nombre d'officiers, elle en prive quelques-uns de profits autrefois plus substantiels et les soumet à une discipline nouvelle. Cette verticalisation s'accompagne d'une reprise en main générale des forces armées. Ce Corps autrefois ouvert aux luttes d'influence et à la pratique des négociations au sein de l'état-major se voit soumis à l'autorité d'un homme qui respecte peu ou pas les principes hiérarchiques, élimine par le limogeage et la suppression de tout avantage financier ses opposants, et n'hésite pas à menacer de mort ceux que ces mesures ne suffisent pas à plier comme en témoigne le sort fait à Diaz Herrera, un ancien proche de Torrijos. On discerne un phénomène analogue au sein des milieux politiques vivant en symbiose avec les forces armées car ces transactions tendent aussi à disparaître au profit d'une "imposición". Ainsi le prédécesseur de Noriega à la tête des forces armées qui avait négocié son départ en échange du soutien du PRD lors de la campagne électorale de 1984, se voit purement et simplement lâché tandis que le candidat du PRD, Nicolas Barletta n'est pas coopté mais imposé par Noriega. Les tensions auront toutes les occasions de se multiplier avec les promotions-limogeages qui seront de règle chez les présidents de la République succédant à Nicolas Barletta, jugé trop indocile.

Derniers lieux d'instabilité, les relations avec les multiples concurrents pour le pouvoir. Ceux-ci sont en effet invités à comprendre que l'heure n'est plus à la négociation, au besoin assortie de démonstration de force à l'intention de chacun des rivaux, ni même à l'exclusion de tel ou tel concurrent en situation de faiblesse, mais bel et bien à la réalisation du gouvernement d'un seul. A cet égard, les gestes de la nébuleuse Noriega ne manquent pas. En 1984, la fraude électorale s'accompagne de multiples violences policières à l'égard des candidats de l'opposition et de leurs supporters. En 1985, un ancien ministre de la Santé de Noriega, vétéran de la lutte armée en Afrique aux côtés d'Amilcar Cabral, puis de Pastora contre Somoza et enfin des Indiens miskitos contre les Sandinistes, est assassiné d'une manière particulièrement atroce après avoir dénoncé la collusion de Noriega avec les trafiquants de drogue et les malversations à la 
tête de l'État panaméen. Par la suite toutes les manifestations de la Croisade civique lancée par le multiples partis de l'opposition seront à leur tour réprimées avec la plus grande brutalité. Enfin, la campagne électorale, préalable au scrutin des élections de 1985 sera l'occasion de violences qui culmineront dans une véritable tentative de lynchage d'un candidat de l'opposition par les hommes de main du régime. A ce propos, il convient de rappeler qu'eu égard aux moeurs politiques panaméennes, l'attitude de Noriega et des forces armées n'est pas seulement créatrice de tensions mais fait surgir très concrètement le spectre d'une coalition de tout ce que le pays compte d'opposants représentants de la classe entrepreneuriale regroupés au sein du CONEP, des partis panaméens, libéro-démocratique-chrétiens et d'une partie des militaires - visant, non plus à tenter de négocier avec Noriega, mais à l'exclure du cercle des concurrents pour le pouvoir. Ainsi, à la différence de Torrijos dont l'utilisation des ressources procurées par les trafics des forces armées a permis un renforcement de ses capacités de production propre ainsi que celle de la Garde nationale, sans pour autant donner lieu à l'émergence de nouveaux champs de conflits, sous Noriega les trafics des forces armées et l'usage de ces ressources ont entraîné l'apparition d'une conflictualité multiple, dans le champ des relations américano-panaméennes, au sein des forces armées, et dans le rapport de celle-ci à la société panaméenne.

De l'émergence d'un nouveau contexte à Just Cause

31 L'apparition de nouveaux lieux de tension, coextensive à la montée en puissance de Noriega, ne correspond pas seulement à l'émergence de tensions spécifiques et circonscrites au domaine des forces armées, des relations panamo-américaines et des rapports entre concurrents pour le pouvoir, mais à l'instauration d'un nouveau contexte marqué par la "désectorisation" de ces domaines et l'entrée en interaction des conflits jadis propres à chacun d'entre eux2'. Décrire les formes prises par cette désectorisation et les interactions entre ces tensions afin de saisir comment ces phénomènes ont créé un contexte favorisant l'opération Just Cause, est le propos de cette dernière partie.

Cette désectorisation semble décelable à deux niveaux : l'un plus structurel renvoie à la place prise par Noriega; l'autre plus conjoncturel s'articule à l'intensification des mobilisations propres à chaque arène.

Les ressources procurées par les trafics menés sous son contrôle n'ont pas seulement procuré à Noriega des ressources de pouvoir supérieures à celles de ces multiples concurrents, mais elles ont fait de lui un personnage qui incarne les tensions propres à ce nouvel état de fait. Ainsi la verticalisation des trafics et sa reprise en main des FI)P, n'ont pas seulement fourni des motifs à la grogne de certains de ses subordonnés ou de ses pairs, mais ces deux opérations ont conduit ses opposants à faire porter sur sa personne tous leurs griefs. On discerne un phénomène analogue au niveau de la société panaméenne. Les rancoeurs des multiples concurrents pour le pouvoir ne sont pas dirigées contre une institution impersonnelle dont Noriega serait le commandant en chef, elles visent au contraire un homme qui se trouve être le commandant en chef des forces armées. Les relations panamo-américaines passent aussi par le même prisme. La République impériale ne fait pas reproche aux Panaméens d'avoir accepté les trafics des militaires et de nombre d'entre eux comme un mode légitime de faire fortune mais dénonce le rôle de l'un d'entre eux dans ces opérations et lui attribue l'entière responsabilité de ces trafics. Cette triple situation ouvre donc logiquement la voie à une interaction entre des tensions, à l'origine circonscrites à des lieux bien particuliers. 

à la retraite par Noriega qui voit dans ce parent de Torrijos un homme capable de lui succéder, Diaz Herrera, le chef d'état- major des FDP, dénonce publiquement la responsabilité de Noriega dans l'assassinat de Hugo Spadafora, dans le trafic d'armes, ainsi que dans la conduite dictatoriale de son gouvernement. Cette mise en cause du rôle de Noriega constitue bel et bien une première, dans la mesure où les conflits internes à une arène, la lutte pour la suprématie au sein des forces armées, amène l'un des acteurs à mettre en liaison ces conflits avec ceux entre concurrents pour le pouvoir et ceux entre Panama et les États-Unis. Dès lors, les différentes entités en conflit avec Noriega ne vont pas seulement passer des alliances ponctuelles (ou de fait) entre elles durant le premier semestre 1988, certains responsables de la Croisade civique appellent de leurs voeux une intervention américaine pour rétablir la démocratie; en octobre 1989 un groupe de putschistes demandent leur soutien aux forces armées des ÉtatsUnis stationnées dans la Canal zone - mais on constate avant tout un désenclavement des espaces de confrontation; désenclavements lisibles dans la chronologie même des événements.

\section{CHRONOLOGIE}

35

- 2 juin 1987 : conférence de presse de Diaz Herrera dénonçant le rôle de Noriega.

- Mi juin 1987: les différents groupes d'opposition lancent une Croisade civique nationale demandant pêle-mêle, "la démocratie, la liberté, la fin de la corruption de la caste au pouvoir".

- 30 juin 1987 : le sous-secrétaire d'État nord-américain E. Abrahams affirme que "l'aire de la complaisance vis-à-vis de la domination des FDP sur la politique panaméenne est terminée $\mathrm{n}$ et suite à ce discours les États-Unis suspendent leur aide annuelle à Panama (26 millions de dollars).

38

- Janvier 1988 : les Etats-Unis proposent sans succès à Noriega d'abandonner le pouvoir, d'organiser les élections libres, et s'engagent en contrepartie à faire lever les charges qui pèsent contre lui.

39

- Février 1988 : Noriega est inculpé de trafic de drogue aux Etats-Unis.

40

- 25 février 1988 : Noriega est déposé par le président Delvalle qui le remplace par son chef d'état-major. L'assemblée dominée par les fidèles de Noriega annule les décisions du président et démet ce dernier de ses fonctions.

41

- Début mars 1988: les Etats-Unis gèlent tous les avoirs du gouvernement panaméen déposés dans les banques américaines.

42

- 16 mars 1988: une partie des forces armées fomentent sans succès un coup d'État pour renverser Noriega. 
43

- Mai 1988: le département d'État propose un nouvel arrangement à l'amiable à Noriega, ce toujours sans succès.

44

- Mai 1988 : Noriega forme un Conseil stratégique militaire chargé d'enquêter sur tous les "actes de trahison" commis par les membres des FDP

45

- Juillet 1988 : Ronald Reagan autorise la CIA à monter des opérations visant à éliminer Noriega.

46

- octobre 1988 : l'Église condamne les violations des Droits de l'homme commises par les forces armées.

47

- 7 Mai 1989 : élection générale.

48

- 10 mai 1989 : les élections remportées par l'opposition sont annulées; au cours d'une manifestation de protestation, les candidats de l'opposition à la présidence - Guillermo Endara - et à la vice-présidence - Guillermo Ford manquent d'être assassinés par les membres d'organisations paramilitaires -les bataillons de la dignité - liées aux forces armées.

49

- 12 mai 1989 : les forces américaines stationnées à Panama organisent des manoeuvres destinées à tester, premièrement leurs capacités à protéger les ressortissants américains, deuxièmement à opérer un débarquement à Panama et à prendre le contrôle de la ville.

50

- 17 mai 1989 : l'Organisation des États américains condamne le régime de Noriega pour avoir annulé les élections.

51

- 11 août 1989 : incidents entre les forces armées américaines et les FDP ; organisations de manoeuvres des FDP baptisées : Fuera el invador - dehors l'envahisseur.

52

- Fin août 1989 : l'OEA appelle de nouvelles élections à Panama.

53

- 31 août 1989 : le Conseil d'État panaméen nomme un président chargé de former un gouvernement provisoire, annonce la dissolution de l'Assemblée nationale et son remplacement par une commission nationale législative, et promet que de nouvelles élections auront lieu dans les six mois.

54

- Septembre 1989: les Etats-Unis suspendent leurs relations diplomatiques avec Panama.

55

- 30 octobre 1989: après s'être concertés avec les Etats-Unis, un groupe d'officiers se soulèvent contre Noriega, engagent de très violents combats contre ses partisans avant d'être défaits puis exécutés. 
- Décembre 1989 : Noriega déclare que le Panama est "en état de guerre avec les EtatsUnis" - selon le journal Libération du 20 décembre 1989.

57

- 16 décembre 1989 : un militaire américain est assassiné par des soldats panaméens, et plusieurs autres sont sérieusement molestés.

58

- 20/31décembre 1989: 24000 Marines interviennent dans le cadre de l'opération Just Cause.

Cette chronologie révèle ainsi que l'on n'assiste pas seulement à un effet cumulatif des événements tout au long de la crise - un durcissement de l'attitude des Etats-Unis, de celle de l'opposition et de celle de Noriega et ses partisans - mais qu'un événement survenu dans une arène se répercute immédiatement dans une autre comme en témoigne le cours pris par les événements en juin 1987, en février-mars 1988, mai 1989, août-septembre 1989 et octobre-décembre 1989.

60 Ainsi en juin 1987, la conférence de presse tenue par Diaz Herrera va être immédiatement utilisée par l'opposition civile, tandis que les Etats-Unis, qui avaient à plusieurs reprises (1941, 1968 et 1984) encouragé ou toléré que les militaires renversent. Cette figure de proue de l'opposition que fut Arnulfo Arias, vont tenir un langage nouveau. Ils appellent à une professionnalisation des forces armées et à l'établissement d'un régime démocratique. Les événements de février-mars 1988 témoignent à la fois d'un processus de désectorisation des conflits et de l'autonomisation de certains acteurs autrefois pris dans des alliances stables. L'inculpation de Noriega par un jury nord-américain conduit un acteur panaméen à tabler sur la fragilisation du commandant en chef des forces armées pour le démettre. Mais il y a plus, car, ce faisant, cet acteur remet en question la coalition entre membres du PRD, des FDP et Noriega, et l'on constate de multiples mouvements d'éclatement dont on connaît au moins deux manifestations. Ainsi malgré l'insuccès de sa première tentative, Delvalle persiste dans sa volonté d'incarner un pôle de pouvoir légitime et autonome vis-à-vis de Noriega et voit ce statut reconnu par les Américains. Par ailleurs, profitant de cette conjoncture un groupe de militaires tente de renverser Noriega. De même en mai 1989, les gestes des différents acteurs témoignent bien d'une interaction entre les multiples champs de conflits. Les démonstrations militaires des États-Unis vont bien évidemment apparaître aux différents protagonistes de la crise panaméenne comme une réponse à l'annulation des élections et à la tentative de lynchage des dirigeants de l'opposition. Les mesures prises par les Etats-Unis en septembre seront perçues de la même façon. Enfin pour nombre de Panaméens (noriéguistes et antinoriéguistes) et d'Américains, l'échec de la tentative de Giroldi ne pouvait déboucher que sur un constat: l'impossibilité de renverser Noriega sans une invasion nord-américaine.

61 Contrairement à ce qu'ont avancé certains commentateurs de la crise panaméenne(24), ces phénomènes d'interaction ne conduisent ni à une fusion des conflits dont témoignerait un repositionnement des acteurs en fonction d'un clivage ami-ennemi, ni un processus de "radicalisation" des différents groupes en présence, processus qui porterait les uns ou les autres à des solutions irréversib1es(25). En effet, quelles que soient les connivences de fait ou les alliances passagères entre certains acteurs, on ne constate aucun repositionnement général de ceux-ci. Les points de vue des forces en 
présence évoluent peu. Les opposants civils s'opposent à Noriega et aux militaires au nom des règles tacites qui président au fonctionnement du système des concurrents pour le pouvoir. Les Etats-Unis mettent en avant les impératifs stratégiques liés au contrôle du Canal, la nécessaire "redémocratisation" et leur volonté de lutter contre le trafic de drogue. Les différents groupes de militaires putschistes visent avant tout à préserver leurs avantages catégoriels mis en danger par la surenchère pratiquée par Noriega. De plus, opposants politiques, militaires et représentants des Etats-Unis divergent en permanence sur l'opportunité de tel ou tel mode d'action. Ainsi, lorsque certains membres de la Croisade civique appelleront de leurs voeux une intervention des Etats-Unis, ceux-ci feront la sourde oreille et seront un peu plus tard tout aussi incapable de soutenir réellement Giroldi et ses compagnons, ce malgré des engagements en ce sens. Et si les points de vue des différents acteurs ont évolué, ces changements ne conduiront jamais à l'apparition d'une grille de lecture commune à l'ensemble des protagonistes. En bref, on n'a semble-t-il aucun exemple de l'élaboration d'une stratégie ou de tactique commune aux différents groupes souhaitant le départ de Noriega.

Par ailleurs, si les événements survenus en juin 1987 marquent bien l'ouverture de la crise panaméenne, en ceci qu'ils entraînent une véritable délégitimation du rôle de Noriega au sein des forces armées sur la scène politique panaméenne et un effondrement des transactions collusives avec les Etats-Unis, ces phénomènes ne sont en rien continus et cumulatifs. La DC, un temps favorable à un coup de force américain, se montre par la suite beaucoup plus réservée à ce sujet tandis que les Panaménéistes auront un cheminement inverse. Les différents groupes de militaires rebelles sont eux aussi porteurs de projets les plus divers au-delà de leur commune volonté de préserver des avantages catégoriels. S'il semble qu'en 1988 ceux-ci aient pu s'accommoder d'une professionnalisation des forces armées, donc qu'un retour aux casernes, il est en revanche attesté(26) que Giroldi et ses pairs n'ont eu qu'un rêve, retrouver un rôle de primus inter pares au sein des concurrents pour le pouvoir à la manière de Torrijos. L'attitude des Etats-Unis n'est en rien différente de celle de la plupart des autres protagonistes. Jusqu'en septembre 1989, et ce, malgré l'inculpation de Noriega par la justice américaine, les Etats-Unis oscilleront entre des solutions allant de l'arrangement direct avec Noriega au coup de force. Notons toutefois que les seuls à faire preuve de continuité dans leurs visées sont Noriega et ses plus proches partisans. Ceux-ci ne révisent à aucun moment leurs objectifs à la baisse, mieux ils semblent rapidement pris dans une logique du quitte ou double comme en témoignent leur attitude à partir de mai 1988.

63 Ainsi convient-il de saisir à la fois comment les tensions coextensives à la montée en puissance de Noriega vont entrer en interaction et comment le contexte de crise créé par celle-ci vont rendre possible l'intervention américaine et d'autre part comment la dé-légitimation de la domination de Noriega sur les FDP et la société panaméenne n'est pas le résultat d'un processus univoque et cumulatif. C'est dire que, quelles que soient les proclamations de Georges Bush aux motifs et aux visées de l'intervention américaine, Just Cause témoigne aussi de l'adaptation à un contexte marqué par deux types de problèmes : d'une part l'impossibilité de négocier avec Noriega, qui refuse un accommodement où les deux parties accepteraient de réviser à la baisse leurs prétentions. D'autre part des problèmes ponctuels, comme la "déclaration de guerre" de Noriega aux Etats-Unis, l'assassinat d'un soldat américain, et les menaces proférées à l'encontre de plusieurs autres. Prenant pour point de départ les incontournables 
réalités recouvertes par le trafic de drogue, nous avons donc été amenés à mettre en évidence trois phénomènes: l'inexistence d'un milieu spécifique; l'articulation du trafic de drogue à de multiples transactions à la limite du légal et de l'illégal, et ce faisant, l'impossibilité de distinguer d'un point de vue sociologique entre ces différents types de trafic ; l'acceptation tacite et parfois explicite de ce mode de faire fortune par l'ensemble des acteurs présents sur la scène panaméenne les politiques et les banquiers, les hommes d'affaires et les syndicalistes, les militaires panaméens et les représentants des Etats-Unis. Nous avons par la suite dû nous interroger sur les répercussions de ces trafics au sein de la vie politique panaméenne. La prise en compte des hypothèses formulées par Anderson nous a permis de mettre à jour ce qu'il est convenu d'appeler la crise panaméenne (1987-1989) : crise qui n'était nullement due à une multiplication des ressources liées au narco-trafic, mais à un problème plus spécifiquement politique tenant à la volonté d'un concurrent pour le pouvoir d'éliminer l'ensemble de ceux qui étaient autrefois autant d'associés rivaux. A cet égard, on peut penser que les conséquences de Just Cause sont tout à fait conformes aux moeurs politiques panaméennes mais peut-être moins à certaines des nouvelles orientations de la République impériale vis-à-vis du sous-continent.

En effet, les concurrents étaient les militaires et leur chef de file Noriega, par trop incapables de respecter les règles du jeu de la société panaméenne, ont non seulement été écartés du cercle des négociations, mais détruits du fait de la dissolution des forces armées et de l'emprisonnement de Noriega. Cela dit, sachant que les trafics que nous évoquions n'ont nullement disparu et vu, les grandes réticences du nouveau président de la République Guillermo Endara - à ratifier le traité d'assistance mutuelle légal avec les Etats-Unis, l'hostilité de nombreux Panaméens à celui-ci et la place prépondérante prise par la zone franche et le Centre financier international dans l'économie du pays, il est probable que les trafics aient encore de beaux jours devant eux. Aussi, pour preuve les utilisateurs de ressources dégagées par ces activités acceptent de composer avec leurs concurrents, celles-ci joueront encore longtemps le rôle qui fut le leur sous Torrijos.

\section{RÉSUMÉS}

Partant des réalités recouvertes par le trafic de drogue, on met en valeur trois phénomènes : l'absence d'un milieu spécifique, l'articulation du trafic de drogue à de multiples autres transactions à la limite du légal et de l'illégal et ce faisant l'impossibilité de distinguer d'un point de vue sociologique entre ces différents types de trafic ; l'acceptation tacite et parfois explicite de cette façon de s'enrichir par l'ensemble des Panaméens. Partant des hypothèses formulées par Anderson sur le système politique latino-américain, on s'interroge sur les répercussions de ces trafics sur les pratiques politiques.

Taking the facts implied by drug-trafficking as a starting point, three phenomenona can be emphasised : the absence of a specific environment ; the articulation of drug-trafficking with many other transactions on the limit between legality and illegality, thus making it impossible to 
distinguish, from a sociological point of view, between these two types of traffic ; the tacit, and sometimes explicit, acceptation of this way of getting rich by all Panamanians. In the light of the hypotheses expressed by Anderson concerning the Latin-American political system, one can raise the question of the consequences of this traffic on political policies.

INDEX

Mots-clés : drogue, contrebande et trafic

Index géographique : Amérique centrale

\section{AUTEUR}

\section{GILLES BATAILLON}

Sociologue 